\title{
ARTICLE
}

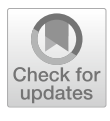

Cite as

Nano-Micro Lett.

(2022) $14: 2$

Received: 29 July 2021

Accepted: 18 October 2021

Published online: 2 December 2021

(C) The Author(s) 2021

\section{Exploring the Spatial Control of Topotactic Phase Transitions Using Vertically Oriented Epitaxial Interfaces}

\author{
Wenrui Zhang ${ }^{1,2}{ }^{凶}$, Jie Zhang ${ }^{1}$, Shaobo Cheng ${ }^{3}$, Christopher M. Rouleau ${ }^{4}$, \\ Kim Kisslinger ${ }^{5}$, Lihua Zhang ${ }^{5}$, Yimei Zhu ${ }^{3}$, Thomas Z. Ward ${ }^{1}$, Gyula Eres ${ }^{1} \bowtie$
}

\section{HIGHLIGHTS}

- An epitaxial nanocomposite approach is developed for exploring spatial control of oxygen vacancy-driven topotactic phase transition of $\mathrm{La}_{0.7} \mathrm{Sr}_{0.3} \mathrm{MnO}_{3-\mathrm{x}}$ (LSMO).

- The ultrahigh density of epitaxial interfaces created in the self-assembled LSMO-NiO nanocomposite films strongly influence the oxygen vacancy formation and topotactic phase distribution in LSMO.

- The distinct intermediate topotactic nanostructures controlled by the $\mathrm{NiO}$ fraction broadens the tuning range of correlated magnetic and transport properties of LSMO.

\begin{abstract}
Engineering oxygen vacancy formation and distribution is a powerful route for controlling the oxygen sublattice evolution that affects diverse functional behavior. The controlling of the oxygen vacancy formation process is particularly important for inducing topotactic phase transitions that occur by transformation of the oxygen sublattice. Here we demonstrate an epitaxial nanocomposite approach for exploring the spatial control of topotactic phase transition from a pristine perovskite phase to an oxygen vacancy-ordered brownmillerite (BM) phase in a model oxide $\mathrm{La}_{0.7} \mathrm{Sr}_{0.3} \mathrm{MnO}_{3}$ (LSMO). Incorporating a minority phase $\mathrm{NiO}$ in LSMO films creates ultrahigh density of vertically aligned epitaxial interfaces that strongly influence the oxygen vacancy formation and distribution in LSMO. Combined structural characterizations reveal strong interactions between NiO and LSMO across the

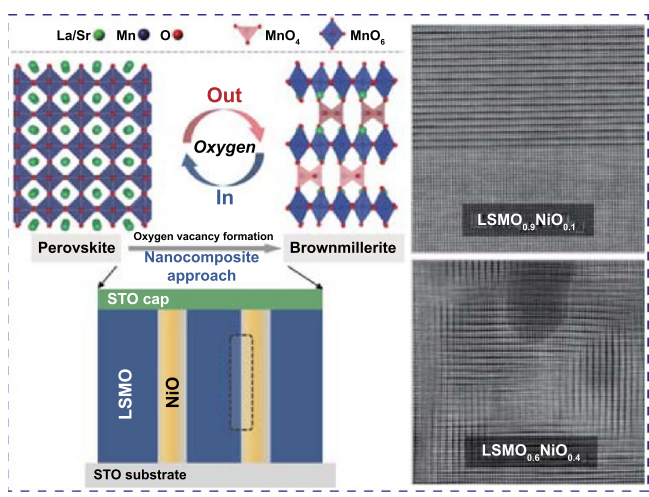
epitaxial interfaces leading to a topotactic phase transition in LSMO accompanied by significant morphology evolution in NiO. Using the $\mathrm{NiO}$ nominal ratio as a single control parameter, we obtain intermediate topotactic nanostructures with distinct distribution of the transformed LSMO-BM phase, which enables systematic tuning of magnetic and electrical transport properties. The use of self-assembled heterostructure interfaces by the epitaxial nanocomposite platform enables more versatile design of topotactic phase structures and correlated functionalities that are sensitive to oxygen vacancies.
\end{abstract}

KEYWORDS Epitaxial interface; Nanocomposite; Functional oxides; Oxygen vacancy; Topotactic phase transition

Wenrui Zhang, zhangwenrui@nimte.ac.cn; Gyula Eres, eresg@ornl.gov

1 Materials Science and Technology Division, Oak Ridge National Laboratory, Oak Ridge, TN 37831, USA

2 Ningbo Institute of Materials Technology and Engineering, Chinese Academy of Sciences, Ningbo, Zhejiang 315201, P. R. China

3 Condensed Matter Physics and Materials Science, Brookhaven National Laboratory, Upton, NY 11973, USA

4 Center for Nanophase Materials Sciences, Oak Ridge National Laboratory, Oak Ridge, TN 37831, USA

5 Center for Functional Nanomaterials, Brookhaven National Laboratory, Upton, NY 11973, USA 


\section{Introduction}

Engineering the oxygen composition is an effective route for creating and modifying a wide range of functionalities for quantum and energy technologies in complex oxides [1-8]. Controlling the formation and distribution of oxygen vacancies has been primarily used for tuning the carrier concentration without changing the parent lattice structure. The variety of methods developed for this purpose includes extrinsic cation doping $[9,10]$, post-synthesis processing $[11,12]$ and heterogeneous boundary formation [13, 14]. At high oxygen vacancy concentrations, a new vacancyordered $\mathrm{ABO}_{2.5}$ brownmillerite (BM) structure forms by a topotactic phase transition that amounts to removing $1 / 6$ of the $\mathrm{O}$ atoms from the $\mathrm{ABO}_{3}$ perovskite (PV) lattice. The $\mathrm{BM}$ structure consists of alternating layers of $\mathrm{BO}_{6}$ octahedra that are apex-linked to oxygen-deficient layers of $\mathrm{BO}_{4}$ tetrahedra. The vacancy-ordered layers form by cooperative interaction of chains created by vacancy clustering that consist of apexlinked $\mathrm{BO}_{4}$ tetrahedra that run along the [110] direction of the perovskite lattice. The oxygen vacancy chains that form between the $\mathrm{BO}_{4}$ chains also run in the [110] direction and are referred to as oxygen vacancy channels (OVCs). These OVCs facilitate fast ion transport in battery electrodes and solid oxide fuel cells $[15,16]$. The PV-to-BM phase transition is observed in a wide range of complex oxides including $\mathrm{SrCoO}_{3-x}[15], \mathrm{SrFeO}_{3-x}[16]$ and $\mathrm{La}_{0.7} \mathrm{Sr}_{0.3} \mathrm{MnO}_{3-x}$ (LSMO) $[17,18]$. The reversible topotactic phase transition between the PV and BM phase is accompanied by a wide range of physical property changes, e.g., from ferromagnetic semimetal in PV-La $\mathrm{L}_{0.7} \mathrm{Sr}_{0.3} \mathrm{MnO}_{3}$ (LSMO-PV) to an antiferromagnetic insulator in $\mathrm{BM}-\mathrm{La}_{0.7} \mathrm{Sr}_{0.3} \mathrm{MnO}_{3-x}$ (LSMO-BM).

The topotactic phase transition provides novel mechanisms for creating a multitude of physical properties from two distinct ground states by stabilizing intermediate metastable phases. Using the topotactic phase transition to achieve desired physical properties requires fine control of the formation dynamics and distribution of oxygen vacancies. The intermediate phase stabilization is especially challenging for manganites since their BM structure is thermodynamically unfavorable $[19,20]$. Previous efforts to modulate the physical properties of manganites by the PVto-BM phase transition have mostly used post-synthesis processing, including vacuum annealing $[17,20]$ and hydrogen plasma treatment [21], or local probe techniques, including direct electron beam scanning [22] and in situ biasing [23], of LSMO-PV films. A pre-synthesis method was also reported effective for controlling the film oxygen content and resulting phases by engineering the substrate oxygen content using pre-growth annealing [24]. The dynamic control of topotactic phase transition has been developed using several methods, such as solid-state electrolyte gating [25, 26], and in situ annealing under different atomspheres [20]. However, the mechanism of the phase transformation and the atomic structure associated with intermediate phases, and their influence on the physical properties require better understanding.

Understanding the spatial evolution of the topotactic phase transition and its influence on the functional responses requires synthesis methods capable of high-level control of oxygen vacancy generation and distribution. The spatial control of topotactic phase transition has not been reported before, but it has been discussed in connection with introducing oxygen vacancy pinning centers into oxide films [27, 28]. We previously reported on the growth of self-assembled LSMO-NiO vertically aligned nanocomposite (VAN) films that feature ultrahigh density of hetero epitaxial vertical interfaces formed between LSMO as a planar matrix and $\mathrm{NiO}$ as a minority phase $[29,30]$. The epitaxial vertical interfaces in the VAN architecture enable effective control of strain-coupled and interface-driven functionalities [31-34]. The LSMO-NiO interfaces were found to modulate the interactions between lattice, spin and chemical composition, which enables exchange-biased magnetoresistance [29] and unusual electric conductivity [30]. Similar LSMO-NiO VAN structures have also been the subject of other studies, which demonstrate perpendicular exchange bias [35] and tunable magnetoresistance [36].

In this study, we take advantage of the strong interaction across the LSMO-NiO interfaces to modulate oxygen vacancy formation and ordering-driven phase transformation in the LSMO. We find that incorporating different fractions of $\mathrm{NiO}$ effectively suppresses oxygen vacancy formation in the LSMO under a fixed vacuum annealing condition, and affects the fraction and the spatial distribution of the resulting BM phase, which strongly correlate with changes in the magnetic and electrical transport properties. For a low $\mathrm{NiO}$ fraction of $x=0.1$, the $\mathrm{NiO}$ is uniformly distributed throughout the LSMO matrix with converted BM phase transformation limited to $38 \%$ volume fraction occurring uniformly near the 
film surface. For a high $\mathrm{NiO}$ fraction of $x=0.4$, the pristine $\mathrm{NiO}$ vertically aligned pillars break up into $\mathrm{NiO}$ nanograins intermixed with different orientations of the BM phase segments and residual unconverted perovskite regions. The magnetization and electrical transport measurements show that the vacuum-annealed nanocomposite films present reversed direction of the change of these properties compared to those of the as-synthesized PV phase films with increasing $x$. These results show that combining topotactic phase transition in LSMO with vertically aligned LSMO-NiO heterostructure interfaces creates novel mechanisms for expanding the tuning range of the properties of LSMO thin films.

\section{Experimental Section}

\subsection{Thin-Film Fabrication}

The (LSMO) $)_{1-x}-(\mathrm{NiO})_{x}$ VAN films with various molar ratios and single-phase LSMO films were deposited on singlecrystal STO (001) substrates at $700{ }^{\circ} \mathrm{C}$ in a dynamic oxygen pressure of 200 mTorr using pulsed laser deposition. The laser fluence was $2 \mathrm{~J} \mathrm{~cm}^{-2}$ and the repetition rate was $5 \mathrm{~Hz}$. The bare films were then cooled down in $200 \mathrm{mTorr}_{2}$ at a cooling rate of $10{ }^{\circ} \mathrm{C} \mathrm{min}^{-1}$. The film thickness was controlled to be $40-50 \mathrm{~nm}$. To grow the oxygen-deficient films, the as-synthesized films were in situ cooled to $600{ }^{\circ} \mathrm{C}$ in a vacuum pressure lower than $5 \times 10^{-6}$ Torr. The STO cap layer was subsequently deposited at a repetition rate of $1 \mathrm{~Hz}$ and with the same laser fluence. The cap layer thickness was around $4 \mathrm{~nm}$. To promote oxygen deficiency, the STOcapped films were in situ annealed in vacuum at $700{ }^{\circ} \mathrm{C}$ for $1 \mathrm{~h}$ and cooled down at a cooling rate of $10{ }^{\circ} \mathrm{C} \mathrm{min}{ }^{-1}$. The nanocomposite film composition was varied by changing the LSMO-to-NiO ratio in the laser ablation targets, which were prepared by established ceramics synthesis techniques.

\subsection{Structural and Chemical Characterization}

High-resolution XRD measurements were taken using a four-circle Panalytical X'pert Pro diffractometer with $\mathrm{Cu}-\mathrm{K} \alpha$ radiation to analyze the film phase and epitaxial growth relations. The film microstructure was investigated with a Hitachi HD2700C microscope in the HAADF mode. Low-magnification STEM/EDX mapping was performed with a FEI Talos $200 \times$ microscope. The cross-sectional
TEM specimens were prepared using a focused ion beam lift-out technique (FEI Helios).

\subsection{Physical Property Measurement}

The thin-film magnetic property measurements, including magnetic hysteresis loops and temperature-dependent magnetization, were taken using a superconducting quantum interference device magnetometer (SQUID, Quantum Design, 7 Tesla). The magnetic field was applied along the in-plane orientation. The substrate background was measured and removed in the presented magnetic data. Electrical transport measurements were taken using a physical property measurement system (PPMS, Quantum Design, 9 Tesla). The film sheet resistance was measured in a typical van der Pauw geometry.

\section{Results and Discussion}

\subsection{Topotactic Phase Tuning via an Epitaxial Nanocomposite Approach}

The process schematic for the reversible perovskite (PV) to brownmillerite (BM) phase transformation in LSMO thin films induced by oxygen vacancy ordering is presented in Fig. 1a. In this section, we use X-ray techniques to determine the effects of the addition of $\mathrm{NiO}$ on the topotactic phase transition in LSMO. The epitaxial (LSMO) $)_{1-x}-(\mathrm{NiO})_{x}$ nanocomposite thin films, typically $40-50 \mathrm{~nm}$ thick, with shorthand notation $\left(\mathrm{L}_{1-x} \mathrm{~N}_{x}\right)$, where $x$ represents the fraction of $\mathrm{NiO}$ in the nanocomposite in 0.1 increments, and LSMO single-phase films were grown on single-crystal $\mathrm{SrTiO}_{3}$ (STO) (001) substrates using pulsed laser deposition (PLD). The oxygen vacancy formation was controlled by vacuum annealing after film growth in combination with a 4-nmthick STO cap layer grown on top of the LSMO and the nanocomposite thin films. This treatment was reported to be an effective method for creating sufficient oxygen vacancies [17, 20], for completing the topotactic PV-to-BM phase transition of single-phase LSMO thin films. The thin films were characterized by X-ray diffraction (XRD) $\theta-2 \theta$ scans, and X-ray reflectivity (XRR) scans are illustrated in Fig. 1b, c. The comparison of the XRD scans of as-grown LSMO$\mathrm{PV}$ thin film and LSMO-BM phase in Fig. S1 clearly shows 

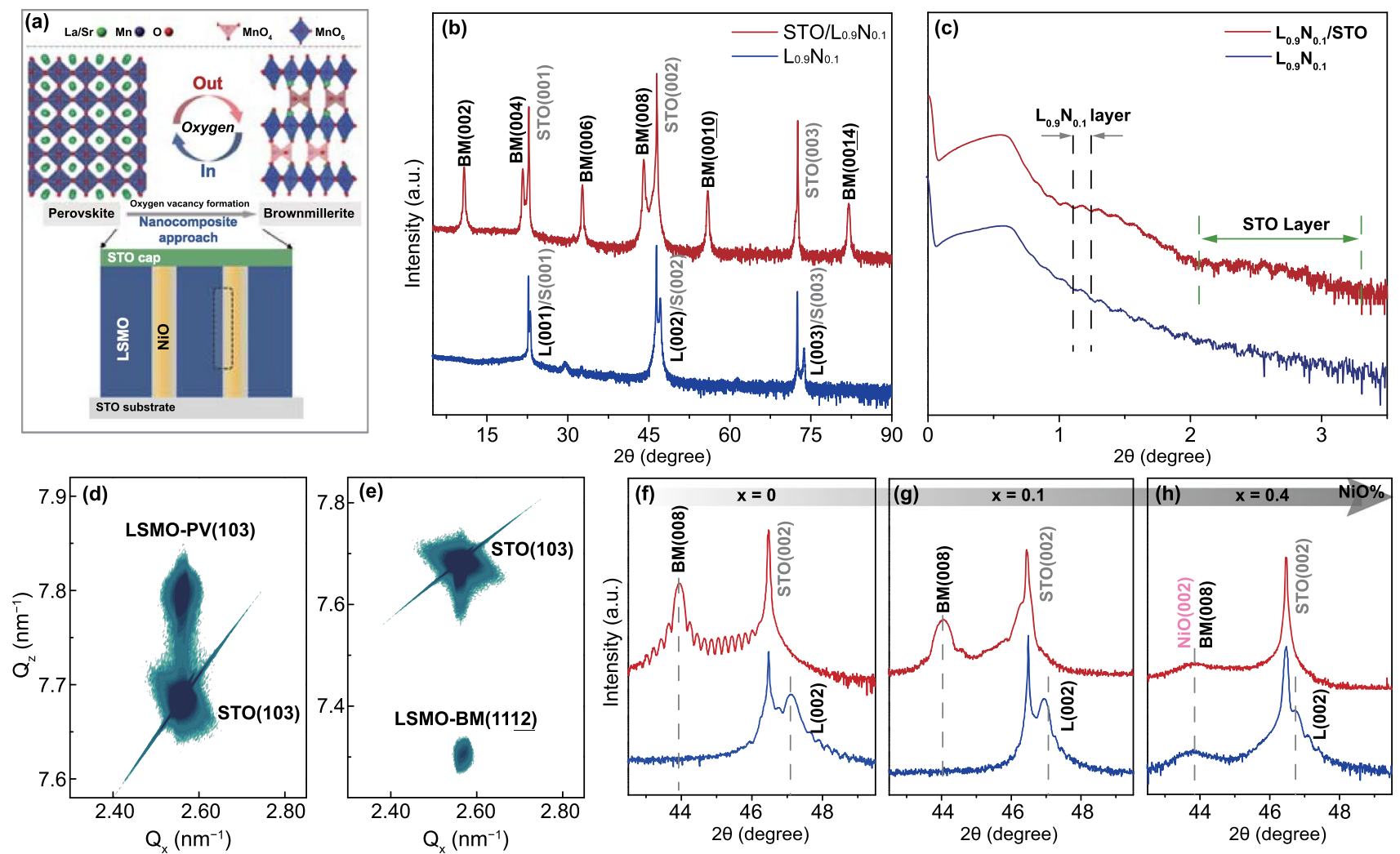

Fig. 1 a Process schematic of the thin-film structure consisting of the vertically oriented NiO nanopillars and STO cap layer with a crystallographic model illustrating the PV-to-BM topotactic phase transformation assisted by oxygen stoichiometry engineering. b $\theta-2 \theta$ XRD scans and $\mathbf{c}$ XRR scans of an as-grown $\mathrm{L}_{0.9} \mathrm{~N}_{0.1}$ film and a vacuum-annealed $\mathrm{STO} / \mathrm{L}_{0.9} \mathrm{~N}_{0.1}$ film. RSM scans near the asymmetric STO (103) diffraction peak for $\mathbf{d}$ an as-grown $\mathrm{L}_{0.9} \mathrm{~N}_{0.1}$ films and $\mathbf{e}$ a vacuum-annealed $\mathrm{STO} / \mathrm{L}_{0.9} \mathrm{~N}_{0.1}$ films. The LSMO peak is given in the orthorhombic notation. $\theta-2 \theta$ XRD local scans of an as-grown PV (blue) films and a vacuum-annealed STO/L $\mathrm{L}_{1-x} \mathrm{~N}_{x}$ film (red) with $\mathbf{f} x=0, \mathbf{g} x=0.1, \mathbf{h} x=0.4$. The film peaks are marked with dashed lines

the appearance of a series of new peaks and the doubling of the unit cell periodicity characteristic of the BM phase. The XRD scan of the $\mathrm{L}_{0.9} \mathrm{~N}_{0.1}$ nanocomposite thin film in Fig. $1 \mathrm{~b}$ shows the same characteristic PV structure of LSMO on STO (100) oriented along the out-of-plane (00 $l$ ) direction. Incorporating $x=0.1$ fraction of $\mathrm{NiO}$ in the nanocomposite film has no observable effect on the LSMO film phase or orientation, with the same set of $\mathrm{PV}(00 l)$ XRD peaks appearing as in the pristine LSMO film. After annealing the $\mathrm{L}_{0.9} \mathrm{~N}_{0.1}$ nanocomposite film with an STO cap layer, the same set of $\mathrm{BM}(00 l)$ peaks appears as in the pure LSMO-BM films, indicating the conversion of the LSMO-PV phase to the BM phase. However, the XRD data do not show whether the PV-to-BM phase transformation is complete and spatially uniform across the film thickness. The role of the $\mathrm{NiO}$ in disrupting the uniformity of the topotactic phase transition is described in the next section by using a local probe such as
STEM imaging. The XRR data for both single-phase LSMO in Fig. S1b and nanocomposite films in Fig. 1c are also very similar. Both sets of data show strong periodic interference oscillations indicating excellent film uniformity and smooth surface and interface for the as-grown $\mathrm{PV} \mathrm{L}_{0.9} \mathrm{~N}_{0.1}$ films and annealed $\mathrm{STO} / \mathrm{L}_{0.9} \mathrm{~N}_{0.1} / \mathrm{STO}(100)$ after the $\mathrm{BM}$ phase transition. The XRR scans for the nanocomposite films in Fig. 1c clearly show a beating pattern in the $\mathrm{STO} / \mathrm{L}_{0.9} \mathrm{~N}_{0.1}$ film that comes from the interference between the $50 \mathrm{~nm}$ LSMO-NiO film and the $4 \mathrm{~nm}$ STO cap layer that is absent in the film with no STO cap layer.

The epitaxial relationship between the STO (100) substrate, and the LSMO and the nanocomposite thin films, respectively, is determined using reciprocal space mapping (RSM). The RSMs near the asymmetric STO (103) peak present vertical alignment of the film and substrate peaks in Fig. 1d, e, which indicate that both as-grown $\mathrm{L}_{0.9} \mathrm{~N}_{0.1}$ and 
vacuum-annealed $\mathrm{STO} / \mathrm{L}_{0.9} \mathrm{~N}_{0.1}$ films are coherently strained to the substrate. The presence of only a single BM reflection $\mathrm{BM}(11 \underline{12})$ in orthorhombic notation indicates that the OVCs are aligned in plane. The RSM maps reveal that the strain changes from tensile to compressive reflected in the increase of the c-axis out-of-plane lattice parameter from smaller to larger than that for STO across the PV-to-BM phase transition. However, such lattice expansion typically originates from the increasing concentration of oxygen vacancies and accompanies a PV-to-BM phase transformation in pure phase LSMO films and cannot be attributed directly to $\mathrm{NiO}$ in the PV-to-BM phase transition. An indication that the addition of $\mathrm{NiO}$ results in structural changes that produce extra lattice strain can be deduced from the XRD scans as a function of changing $\mathrm{NiO}$ fraction. Adjusting the $\mathrm{NiO}$ fraction ratio $x$ in the nanocomposite films demonstrates apparent influence on the topotactic phase transformation of the constituent phase LSMO. This is evidenced from the systematic XRD peak evolution near the STO $(002)$ in local $\theta-2 \theta$ scans (Figs. 1f-h and S2a, S3). The XRD peaks of LSMO-PV(002), designated as L(002) represented by the blue curve, are clearly seen in as-grown LSMO and LSMO$\mathrm{NiO}$ films. Note a weak $\mathrm{NiO}(002)$ reflection starts appearing for $x \geq 0.4$. After a fixed vacuum annealing treatment, sharp $\mathrm{BM}(002)$ peaks arise for $x \leq 0.2$ and then become broader and disordered for $x \geq 0.4$ (Figs. $1 \mathrm{~h}$ and S3).

Important clues for understanding the cause for the shift and the broadening of both peaks can be obtained from XRD scans for the intermediate stages of the PV-to-BM phase transformation in pure LSMO films that were reported previously [20]. The key feature of the XRD scans is that the L(002) peak shifts toward smaller angles with increasing annealing time that increases the vacancy concentration to eventually move to the lower side of the STO (002) peak. After this happens, the $\mathrm{BM}(008)$ peak starts growing and the $\mathrm{L}(002)$ peak gradually loses intensity and disappears when the PV-to-BM phase transformation is complete. In this study, the annealing time was kept fixed and determined to be sufficiently long to allow completion of the PV-to-BM phase transition. As $x$ increases from 0 to 0.4 , the $\mathrm{L}(002)$ peak shifts toward smaller angles indicating increasing strain in the as-grown films and becomes broader suggesting increasing disorder. Meanwhile, a noticeable peak shoulder near the STO (002) reflection arises for $\mathrm{L}_{0.9} \mathrm{~N}_{0.1}$, which is related to the remaining oxygen-deficient PV phase. In nanocomposite films with completed PV-to-BM transformation the $\mathrm{BM}(008)$ peak also becomes weaker and broader with increasing $x$. There are two important interpretations of these observations. First is that the NiO effectively suppresses oxygen vacancy formation and hinders the PV-to$\mathrm{BM}$ phase transformation. The second is that $\mathrm{NiO}$ stabilizes distinct disordered microstructures during the topotactic phase transition process. The STEM imaging data and the discussion in the following section shows that these two mechanisms are intertwined and not straightforward to separate unambiguously.

\subsection{Microstructure of Topotactically Intermediate Phases}

The evolution of the topotactic phase and the characterization of the local structure created by the topotactic phase transformation of the vacuum-annealed STO/L ${ }_{1-x} \mathrm{~N}_{x}$ I STO(100) films as a function of $x$ was performed by STEM imaging in the high-angle annular dark-field (HAADF) mode. We start with the low-magnification cross-sectional STEM image in Fig. 2a that shows the $\mathrm{L}_{0.9} \mathrm{~N}_{0.1}$ film consisting of a bright phase contrast region sandwiched between two darker contrast regions consisting of the STO cap layer on the top and the STO substrate on the bottom. A continuous horizontal structural boundary marked by white dashed arrows separating the top and the bottom regions of the film is clearly visible through the middle of the film. The energy-dispersive X-ray spectroscopy (EDS) maps in Fig. 2b reveal that $\mathrm{NiO}$ is homogeneously distributed in the film, and show no observable variation in the distribution of the other elements between the top and bottom regions of the film. However, the high-resolution STEM image in Fig. 2c reveals that the vacuum-annealed $\mathrm{STO} / \mathrm{L}_{0.9} \mathrm{~N}_{0.1}$ film actually consists of three regions, two $\mathrm{PV}$ phase regions, one at the bottom starting at the STO substrate-film interface that is $\sim 28 \mathrm{~nm}$ thick and the other at the top starting at the STO cap-film interface that is $\sim 4 \mathrm{~nm}$ thick and a homogeneous BM phase in the middle region with a thickness of $\sim 20 \mathrm{~nm}$. The oxygen vacancy channels in the BM phase run along the in-plane film direction, as featured by the periodic alignment of the dark contrast plane sandwiched by two brighter atom planes. This corroborates the $[00 l]$ axis as the out-of-plane orientation observed from the XRD result. The uniform distribution of the oxygen-deficient layers in Fig. 2c allows an estimate of the volume fraction of the LSMO lattice that is 


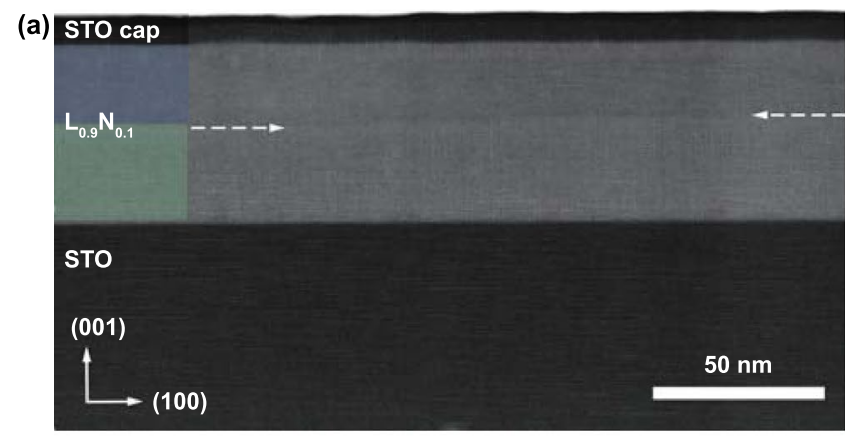

(b)
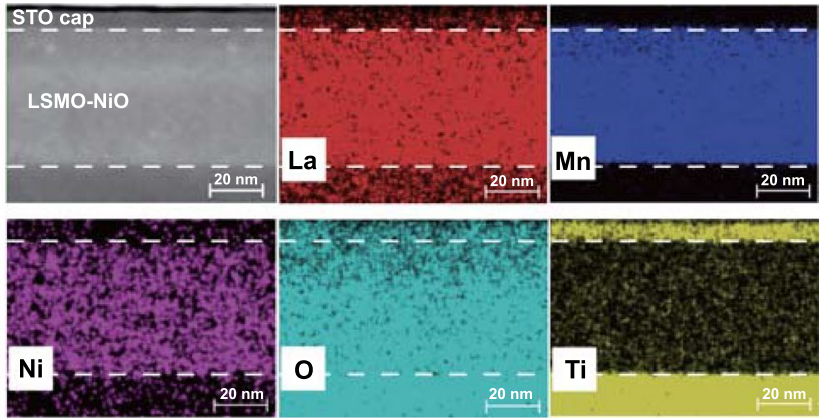

(c)
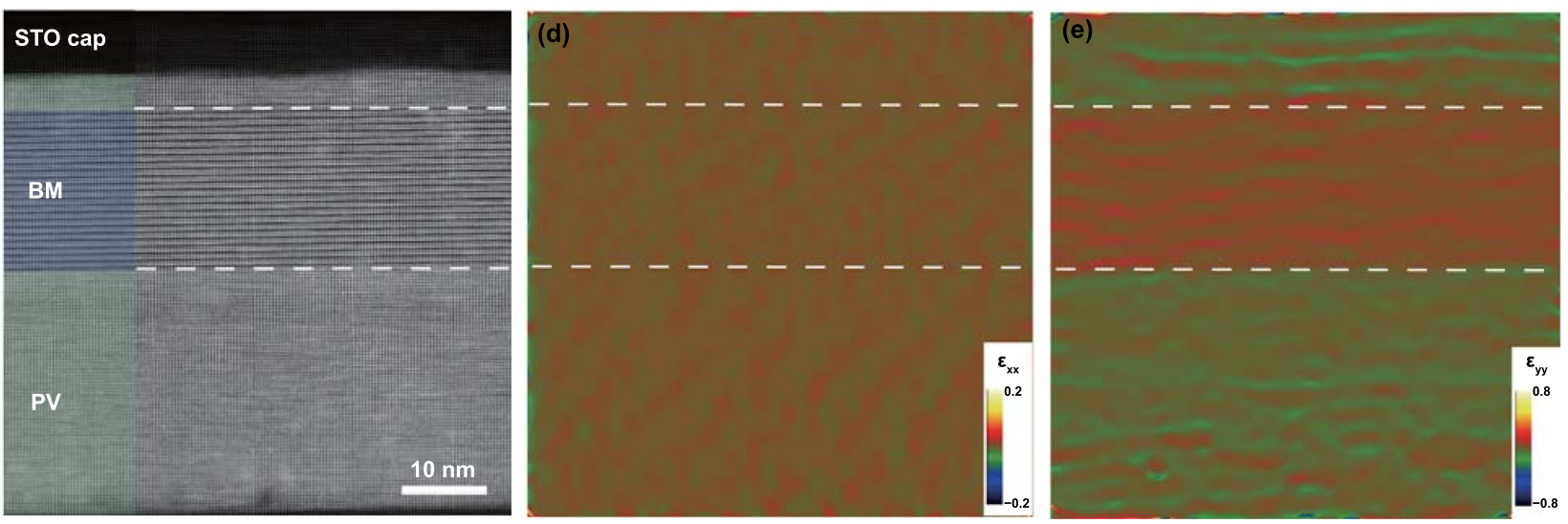

Fig. 2 a Cross-sectional HAADF-STEM image and $\mathbf{b}$ element-specific EDS maps of a vacuum-annealed STO/L ${ }_{0.9} \mathrm{~N}_{0.1}$ film illustrating the overall thin-film structure and uniform distribution Ni and the constituent elements of the film. c High-resolution STEM image showing a three-layer structure formed in the vacuum-annealed $\mathrm{STO} / \mathrm{L}_{0.9} \mathrm{~N}_{0.1}$ film consisting of a 4-nm-thick PV phase as the top layer, a 20-nm-thick BM phase as the middle layer and a 28 -nm-thick PV phase as the bottom layer. The corresponding strain maps along the $\mathbf{d} \varepsilon_{\mathrm{xx}}$ and $\mathbf{e} \varepsilon_{\mathrm{yy}}$ directions. The phase boundaries between PV and BM structures are marked with white dotted lines. The entire bottom PV region is used as the reference for the GPA analysis

converted to the $\mathrm{BM}$ phase. Using the total film thickness as $4+28+20=52 \mathrm{~nm}$ and the thickness of the BM layer of $20 \mathrm{~nm}$ roughly gives $20 / 52=0.38$ for the fraction of the converted phase. This estimate of partial conversion is in agreement with the XRD data in Fig. 1g that show appearance of a shoulder corresponding to broadening by the $\mathrm{L}(002)$ perovskite peak indicating the presence of a detectable fraction of the perovskite phase.

The observation of three regions in vacuum-annealed $\mathrm{STO} / \mathrm{L}_{0.9} \mathrm{~N}_{0.1}$ film is in sharp contrast with the single-phase STO/LSMO film that is fully converted to a pure BM phase based on XRD and STEM data after the same annealing treatment. This is a quite unexpected and intriguing result because the STO cap layer is believed to be the oxygen vacancy source for driving the topotactic phase transformation, and based on that, one would expect that the BM phase would start (nucleate) at the STO cap-film interface. The possibility that this distinct phase ordering is related to strain distribution throughout the $\mathrm{STO} / \mathrm{L}_{0.9} \mathrm{~N}_{0.1}$ film. The geometric phase analysis (GPA) method is further exploited, which shows that the distribution of strain distortion largely follows the phase structure evolution. It is observed a relatively uniform strain distribution along the in-plane (IP) $\varepsilon_{\mathrm{xx}}$ direction in both the PV and BM phases in Fig. 2d. The strain modulation along the out-of-plane (OP) $\varepsilon_{\mathrm{yy}}$ direction appears larger in the BM phase than the PV phases as shown in Fig. 2e. The upper PV region shows slightly larger strain modulation than the bottom region, which is likely due to a process artifact [37], since the bottom PV region is used as a reference for the GPA analysis. A corresponding line profile is provided in Fig. S5, which is consistent with the phase evolution and the alignment of oxygen vacancy channels.

Increasing the $\mathrm{NiO}$ concentration has a dramatic effect on the outcome of the topotactic phase transition process and forms completely different intermediate structure illustrated by the example of the $\mathrm{STO} / \mathrm{L}_{0.6} \mathrm{~N}_{0.4}$ film. The 
low-magnification STEM image in Fig. 3a and the EDS maps in Fig. $3 b$ show that after the vacuum annealing step the $\mathrm{NiO}$ is distributed randomly throughout the LSMO matrix in form of $\mathrm{NiO}$ nanograin particles. In the as-grown $\mathrm{L}_{0.6} \mathrm{~N}_{0.4}$ film, the $\mathrm{NiO}$ self-segregates into ultrafine vertically aligned nanopillars that are uniformly embedded in a planar LSMO matrix shown in Fig. S4. Atomic resolution STEM image in Fig. 3c shows that LSMO maintains the PV structure and forms continuous vertical interfaces with $\mathrm{NiO}$ nanopillars throughout the film thickness. Atomic resolution STEM images in Fig. 3d, e show that after the annealing step the nanopillars break up into $\mathrm{NiO}$ nanograin particles randomly distributed throughout the LSMO matrix that is also reorganized into vertically and horizontally oriented
BM phase segments, and a small amount of residual PV segments also remains (Fig. S6). The structural evolution of $\mathrm{NiO}$ from cylindrical to spherical shape could be a path for reducing the interface energy during the vacuum annealing process. This shape evolution, however, is somewhat surprising, considering that previous reports consider the nanopillars to be rather robust once formed within the LSMO matrix. This is quite different from the $\mathrm{L}_{0.9} \mathrm{~N}_{0.1}$ film that shows uniform distribution of the $\mathrm{NiO}$ in the film and uniform layers of the c-axis oriented BM phase in the top part of the film. The interface strain coupling is likely a primary factor for the observed structural evolution as a function of the $\mathrm{NiO}$ fraction. The strain originates from the large lattice mismatch between LSMO and $\mathrm{NiO}$ estimated from the bulk (a)

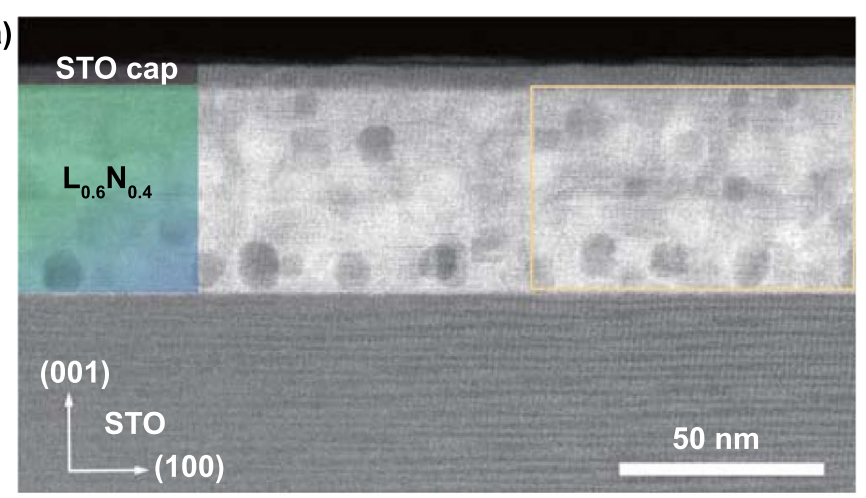

(c)

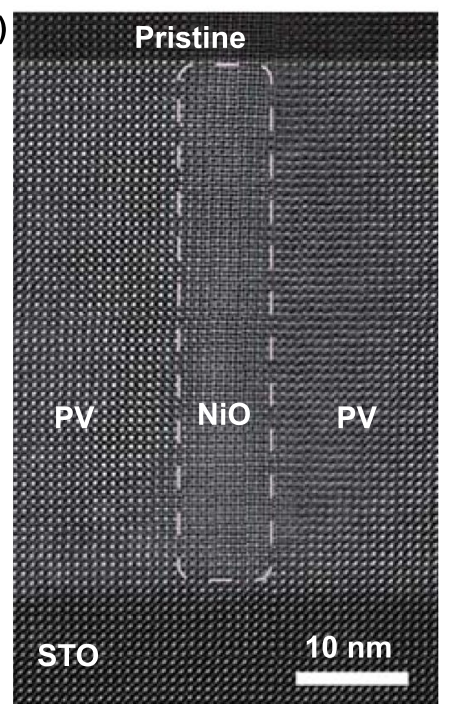

(d)

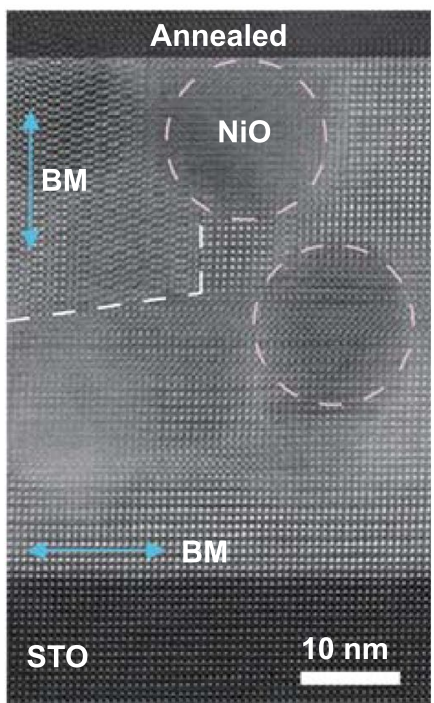

(b)

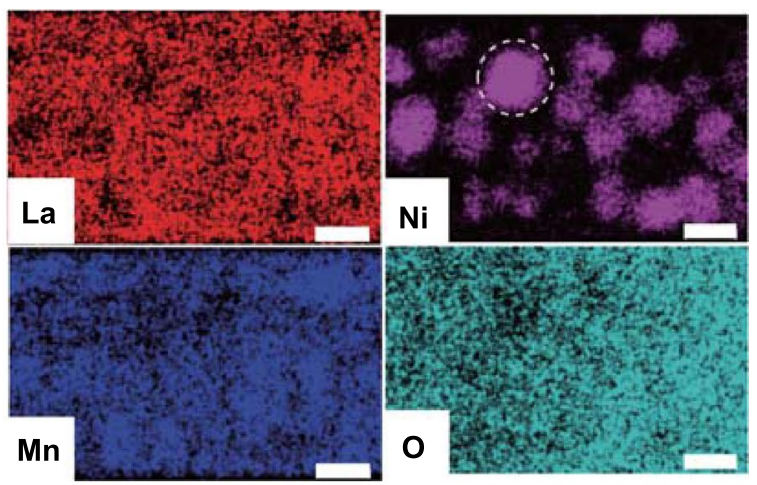

(e)

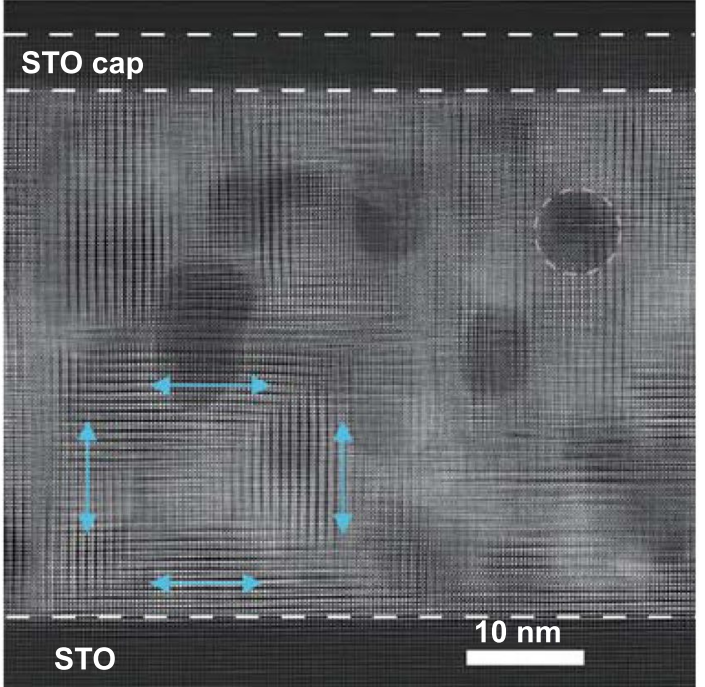

Fig. 3 a Cross-sectional low-magnification HAADF-STEM image and b element-specific EDS maps of a vacuum-annealed STO/L ${ }_{0.6} \mathrm{~N}_{0.4}$ film identifying the formation of $\mathrm{NiO}$ nanograins distributed throughout the film. Atomic resolution STEM images of $\mathbf{c}$ as-grown PV $\mathrm{L}_{0.6} \mathrm{~N}_{0.4}$ film showing a vertical $\mathrm{NiO}$ nanopillar running through the $\mathrm{PV}$ film and d vacuum-annealed STO/L ${ }_{0.6} \mathrm{~N}_{0.4}$ film showing $\mathrm{NiO}$ nanograins, BM phase segments with perpendicular OVC directions indicated by the light blue arrows and residual PV phase segments. e STEM image divided into several regions by white dashed lines. The orientations of BM domains are marked by light blue arrows, and the shape of NiO is highlighted by pink dashed circles 
lattice constants of $\mathrm{c}_{\mathrm{LSMO}}=0.387 \mathrm{~nm}$ and $\mathrm{c}_{\mathrm{NiO}}=0.417 \mathrm{~nm}$ to be $\sim 7.7 \%$, with further details about the strain in LSMO$\mathrm{NiO}$ vertical interfaces given in our previous work $[29,30]$. The chemical information of the interface structure is further analyzed using electron energy loss spectroscopy (EELS) by probing the Mn oxidation state. For the vacuum-annealed $\mathrm{L}_{0.6} \mathrm{~N}_{0.4}$ film, the EELS spectra show no observable difference in the Mn oxidation state at positions near and away from the LSMO-NiO interface (Fig. S7), suggesting that the cation interdiffusion is not significant. This is consistent with the distinct phase segregation as observed by the EDS results in Fig. 3b. The dramatic nanostructure difference between $\mathrm{L}_{0.9} \mathrm{~N}_{0.1}$ and $\mathrm{L}_{0.6} \mathrm{~N}_{0.4}$ motivates us to examine the nanostructure of the vacuum-annealed $\mathrm{STO} / \mathrm{L}_{0.8} \mathrm{~N}_{0.2}$ film, which is an intermediate nanostructure between the above two representative compositions. Interestingly, the STEM image in Fig. S2b shows that the $\mathrm{L}_{0.8} \mathrm{~N}_{0.2}$ film consists of a top BM structure layer and a bottom PV structure layer with small $\mathrm{NiO}$ clusters uniformly distributed throughout the film. Indeed, this unique nanostructure combines the characteristic structural features of both end member films $\mathrm{L}_{0.9} \mathrm{~N}_{0.1}$ and $\mathrm{L}_{0.6} \mathrm{~N}_{0.4}$, thus depicting the full structure evolution range.

\subsection{Systematic Tuning of Magnetic and Transport Characteristics}

The identification of distinct microstructures stabilized by LSMO-NiO films raises the intriguing possibility that the magnetic and electrical transport properties of the nanostructured films can be linked directly to these features by varying the NiO fraction. Figure 4a-c compare the magnetization versus magnetic field curves $(M-H)$ of representative $\mathrm{L}_{1-x} \mathrm{~N}_{x}$ and $\mathrm{STO} / \mathrm{L}_{1-x} \mathrm{~N}_{x}$ films with $x=0,0.1$ and 0.4 measured at $10 \mathrm{~K}$ with an in-plane magnetic field. As the baseline comparison, the complete phase transition in the single-phase LSMO films in Fig. 4a shows a dramatic decrease in the saturation magnetization $\left(M_{\mathrm{s}}\right)$ from $569 \mathrm{emu} \mathrm{cc}^{-1}$ for the PV phase to $38 \mathrm{emu} \mathrm{cc}^{-1}$ for the BM phase, giving a $M_{\mathrm{s}}(\mathrm{STO} /$ $\mathrm{LSMO}) / M_{\mathrm{s}}(\mathrm{LSMO})$ ratio of $6.7 \%$. The temperature-dependent magnetization $(M-T)$ curves in Fig. $4 \mathrm{~d}$ show a decreasing $M_{\mathrm{s}}$ and a decreasing Curie temperature $\left(T_{\mathrm{c}}\right)$ with increasing $x$ for the uncapped nanocomposite films. The nanocomposite films with the STO cap layer after annealing show the opposite, a mildly increasing $M_{\mathrm{s}}$ with increasing $x$. Specifically,
Fig. $4 \mathrm{~d}$ shows that as $x$ increases from 0 to $0.4, M_{\mathrm{s}}$ for $\mathrm{L}_{1-x} \mathrm{~N}_{x}$ films with the LSMO pristine PV phase decreases from 569 to $254 \mathrm{emu} \mathrm{cc}^{-1}$, while $M_{\mathrm{s}}$ for vacuum-annealed STO/L ${ }_{1-x} \mathrm{~N}_{x}$ films increases from $38 \mathrm{emu} \mathrm{cc}^{-1}$ in STO/LSMO to $57 \mathrm{emu}$ $\mathrm{cc}^{-1}$ in $\mathrm{STO} / \mathrm{L}_{0.9} \mathrm{~N}_{0.1}$. For as-grown $\mathrm{L}_{1-x} \mathrm{~N}_{x}$ VAN films, the smaller $M_{\mathrm{s}}$ with larger $x$ is attributed to a number of factors, including the establishment of magnetic exchange coupling with the antiferromagnetic $\mathrm{NiO}$ at the LSMO-NiO interface $[38,39]$, the strain effect imposed by the secondary phase [40] and more point defects in the nanocomposite films [41]. Such phenomenon is usually seen in nanocomposite films including LSMO-MgO, $\mathrm{LSMO}-\mathrm{ZrO}_{2}$ and $\mathrm{LSMO}-\mathrm{ZnO}$ VAN films [42]. As a result, the reduced magnetization is accompanied by larger magnetic coercive field observed in the $M-H$ curves (Fig. $4 \mathrm{~b}, \mathrm{c}$ ) and lower $T_{\mathrm{c}}$ observed in the temperature-dependent magnetization $(M-T)$ curves (Fig. 4d) for NiO-rich VAN films. In contrast, for vacuumannealed STO/L ${ }_{1-x} \mathrm{~N}_{x}$ films, the single-phase LSMO exhibits the lowest $M_{\mathrm{s}}$ because of its phase pure antiferromagnetic BM structure.

The intermediate nanostructures stabilized during the topotactic phase transition exhibit systematic variation of electrical transport characteristics, which are evaluated by temperature-dependent electrical transport measurements. The temperature-dependent sheet resistance $\left(R_{s}-T\right)$ designated by a star on the measurement limit in Fig. $4 \mathrm{e}$ for a complete PV-to-BM transition in LSMO shows a dramatic increase in room-temperature $R_{s}$ by more than five orders of magnitude. In contrast, the range of $R_{S}$ systematically decreases with increasing $x$ in $\mathrm{L}_{1-x} \mathrm{~N}_{x}$ VAN films and becomes less than two orders of magnitudes for the $\mathrm{L}_{0.6} \mathrm{~N}_{0.4}$ film. In contrast, the room-temperature sheet resistance of the nanocomposite films with a cap layer decreases as a function of $x$ after annealing. The room-temperature $M_{\mathrm{s}}$ and $R_{S}$ results as a function of $x$ are summarized in Fig. 4f, g. The schematic illustration of the spatial distribution of different nanostructure configurations in the nanocomposite films as a function of $x$ is shown in Fig. 4h. These summary plots clearly demonstrate that varying the $\mathrm{NiO}$ fraction results in systematic changes of magnetic and electrical transport properties by modulating the topotactic phase transition process. The interpretation of this behavior is provided by the local STEM imaging and characterization, which shows that incorporating $\mathrm{NiO}$ in the nanocomposite films suppresses oxygen vacancy formation in LSMO and provides a potential 

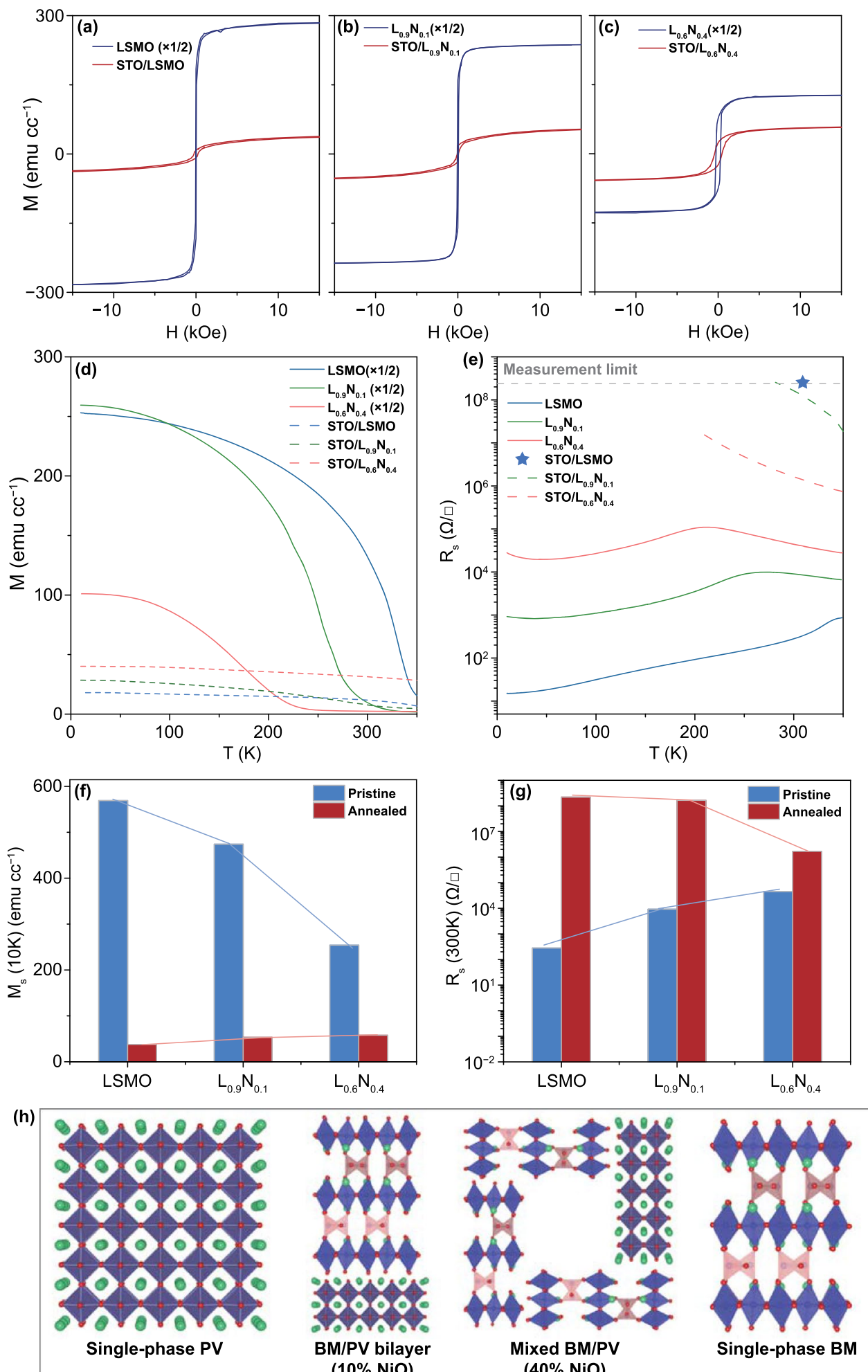

Fig. 4 a-c Magnetic hysteresis curves, d temperature-dependent field-cooled magnetization and e temperature-dependent sheet resistance of as-grown PV and vacuum-annealed BM L ${ }_{1-x} \mathrm{~N}_{x}$ films with $x=0, x=0.1, x=0.4$, the star designates the range for a pure LSMO-BM film The magnetization values of as-grown $\mathrm{L}_{1-x} \mathrm{~N}_{x}$ films are presented after multiplying a factor of $1 / 2$ for better illustration with the much smaller values of vacuum-annealed $\mathrm{BM} \mathrm{L}_{1-x} \mathrm{~N}_{x}$ films. $\mathbf{f}$ Saturation magnetization $\mathrm{M}_{\mathrm{s}}$ at $10 \mathrm{~K}$ and $\mathbf{g}$ film sheet resistance $\mathrm{R}_{\mathrm{s}}$ at $300 \mathrm{~K}$ of as-grown PV and vacuum-annealed $\mathrm{BM} \mathrm{L} \mathrm{L}_{1-x} \mathrm{~N}_{x}$ films as a function of the NiO ratio $x$. The solid lines are used as a guide to the eye to highlight the systematic magnetization and sheet resistance variation as a function of the film composition and phase evolution. $\mathbf{h}$ Schematic illustration of the spatial distribution of different nanostructure configurations in the nanocomposite films as a function of the nominal NiO fraction $x$ 
control mechanism for systematic tuning of BM phase formation and PV phase stabilization.

The combination of structural, imaging and transport data shows that incorporation of heterostructure interfaces by the epitaxial nanocomposite approach provides additional degrees of freedom to modulate spatially the topotactic phase transition and expands the tunability range of correlated functional behavior. This approach is different from previous studies that were mainly focused on postgrowth treatment of single-phase LSMO films [17, 20]. It is intriguing to observe the structural evolution of $\mathrm{NiO}$ from nanopillars to nanograins accompanied by the topotactic phase transition in LSMO. This behavior suggests that the heterostructure interfaces formed by $\mathrm{NiO}$ and LSMO have a more intricate role than just providing an inert phase boundary. Instead, these interfaces play an active role in regulating and coordinating oxygen vacancy migration and distribution to both of the end members. Understanding the fundamental mechanism on how $\mathrm{NiO}$ interacts with oxygen vacancy formation requires further theoretical and experimental study, which will be extremely helpful for guiding the selection and design of other secondary phases to be coupled with LSMO. The use of self-assembled heterostructure interfaces uniformly distributed in LSMO could enable precise control of topotactic phase transition occurring at or away from the interface, thus significantly enhancing the design flexibility for interface-governed functionality. Potential device applications include creation of localized channels for separate electron or ion transport in memory devices and fuel cells, and exploration of effective ways for fine control of magnetism in spintronic devices.

\section{Conclusions}

We demonstrate the use of self-assembled interfaces in the epitaxial nanocomposite platform as an effective route for spatially controlling the PV-to-BM topotactic phase transition in LSMO and systematically tuning the correlated physical properties. The epitaxial interfaces are established by incorporating $\mathrm{NiO}$ as a minority phase with LSMO in self-assembled nanocomposite films, which features ultrahigh density of LSMO-NiO vertically aligned interfaces and strong interface coupling. The epitaxial interfaces are actively involved in modulating the oxygen vacancy formation and distribution in LSMO, which is accompanied by significant morphology evolution in $\mathrm{NiO}$. A simple control parameter, the NiO nominal ratio, is used to stabilize different intermediate topotactic nanostructures that are linked to a multitude of magnetic and electrical transport properties from two distinct ground states. The epitaxial nanocomposite approach for creating self-assembled epitaxial interfaces adds the capability for spatially controlling topotactic phase transitions and enhances the materials design flexibility for emerging quantum information and sensing and advanced energy applications.

Acknowledgements This work was supported by the US Department of Energy (DOE), Office of Science, Basic Energy Sciences (BES), Materials Sciences and Engineering Division. Part of this research was performed at the Center for Nanophase Materials Sciences at Oak Ridge National Laboratory, which is a DOE Office of Science User Facility. The electron microscopy work done at Brookhaven National Laboratory was supported by the U.S. DOEBES, the Center for Functional Nanomaterials, a DOE Office of Science User Facility, and Materials Science and Engineering Division, under Contract No. DE-SC0012704. W. Z. acknowledges the support by National Natural Science Foundation of China (Grant No. 62004200) and Zhejiang Provincial Natural Science Foundation (Grant No. LZ21F040001). S. C. acknowledges the support by Q-MEEN-C, an Energy Frontier Research Center funded by the U.S. DOE-BES under award No. DE-SC0019273.

Funding Open access funding provided by Shanghai Jiao Tong University.

Open Access This article is licensed under a Creative Commons Attribution 4.0 International License, which permits use, sharing, adaptation, distribution and reproduction in any medium or format, as long as you give appropriate credit to the original author(s) and the source, provide a link to the Creative Commons licence, and indicate if changes were made. The images or other third party material in this article are included in the article's Creative Commons licence, unless indicated otherwise in a credit line to the material. If material is not included in the article's Creative Commons licence and your intended use is not permitted by statutory regulation or exceeds the permitted use, you will need to obtain permission directly from the copyright holder. To view a copy of this licence, visit http://creativecommons.org/licenses/by/4.0/.

\section{References}

1. R.J. Cava, B. Batlogg, C.H. Chen, E.A. Rietman, S.M. Zahurak et al., Oxygen stoichiometry, superconductivity and normal-state properties of $\mathrm{YBa}_{2} \mathrm{Cu}_{3} \mathrm{O}_{7-\delta}$. Nature 329, 423-425 (1987). https://doi.org/10.1038/329423a0

2. N. Biškup, J. Salafranca, V. Mehta, M.P. Oxley, Y. Suzuki et al., Insulating ferromagnetic $\mathrm{LaCoO}_{3}$ films: A phase induced 
by ordering of oxygen vacancies. Phys. Rev. Lett. 112, 087202 (2014). https://doi.org/10.1103/PhysRevLett.112.087202

3. W. Zhang, A.R. Mazza, E. Skoropata, D. Mukherjee, B. Musico et al., Applying configurational complexity to the 2D ruddlesden-popper crystal structure. ACS Nano 14, 1303013037 (2020). https://doi.org/10.1021/acsnano.0c04487

4. J.H. Lee, R.H. Shin, W. Jo, Polarization switching and relaxation dynamics of bismuth layered ferroelectric thin films: Role of oxygen defect sites and crystallinity. Phys. Rev. B 84, 094112 (2011). https://doi.org/10.1103/PhysRevB.84.094112

5. Y.-M. Kim, J. He, M.D. Biegalski, H. Ambaye, V. Lauter et al., Probing oxygen vacancy concentration and homogeneity in solid-oxide fuel-cell cathode materials on the subunit-cell level. Nat. Mater. 11, 888-894 (2012). https://doi.org/10.1038/ nmat3393

6. W. Zhang, J.L. Lyons, J. Cen, M.Y. Sfeir, M. Liu, Multicomponent oxynitride thin films: Precise growth control and excited state dynamics. Chem. Mater. 31, 3461-3467 (2019). https:// doi.org/10.1021/acs.chemmater.9b00656

7. M. Kim, B. Lee, H. Ju, J.Y. Kim, J. Kim et al., Oxygenvacancy-introduced $\mathrm{BaSnO}_{3-\delta}$ photoanodes with tunable band structures for efficient solar-driven water splitting. Adv. Mater. 31, 1903316 (2019). https://doi.org/10.1002/adma.201903316

8. W. Zhang, F. Wu, J. Li, D. Yan, J. Tao et al., Unconventional relation between charge transport and photocurrent via boosting small polaron hopping for photoelectrochemical water splitting. ACS Energy Lett. 3, 2232-2239 (2018). https://doi. org/10.1021/acsenergylett.8b01445

9. J. Seidel, W. Luo, S.J. Suresha, P.K. Nguyen, A.S. Lee et al., Prominent electrochromism through vacancy-order melting in a complex oxide. Nat. Commun. 3, 799 (2012). https://doi.org/ 10.1038/ncomms 1799

10. J. Gazquez, S. Bose, M. Sharma, M.A. Torija, S.J. Pennycook et al., Lattice mismatch accommodation via oxygen vacancy ordering in epitaxial $\mathrm{La}_{0.5} \mathrm{Sr}_{0.5} \mathrm{CoO}_{3-\delta}$ thin films. APL Mater. 1, 012105 (2013). https://doi.org/10.1063/1.4809547

11. X. Chen, L. Liu, P.Y. Yu, S.S. Mao, Increasing solar absorption for photocatalysis with black hydrogenated titanium dioxide nanocrystals. Science 331, 746-750 (2011). https://doi. org/10.1126/science. 1200448

12. W. Zhang, L. Song, J. Cen, M. Liu, Mechanistic insights into defect-assisted carrier transport in bismuth vanadate photoanodes. J. Phys. Chem. C 123, 20730-20736 (2019). https://doi. org/10.1021/acs.jpcc.9b04583

13. T. Rojac, A. Bencan, G. Drazic, N. Sakamoto, H. Ursic et al., Domain-wall conduction in ferroelectric $\mathrm{BiFeO}_{3}$ controlled by accumulation of charged defects. Nat. Mater. 16, 322-327 (2017). https://doi.org/10.1038/nmat4799

14. W. Zhang, D. Yan, J. Li, Q. Wu, J. Cen et al., Anomalous conductivity tailored by domain-boundary transport in crystalline bismuth vanadate photoanodes. Chem. Mater. 30, 1677-1685 (2018). https://doi.org/10.1021/acs.chemmater.7b05093

15. H. Jeen, W.S. Choi, M.D. Biegalski, C.M. Folkman, I.C. Tung et al., Reversible redox reactions in an epitaxially stabilized $\mathrm{SrCoO}_{\mathrm{x}}$ oxygen sponge. Nat. Mater. 12, 1057-1063 (2013). https://doi.org/10.1038/nmat3736
16. A. Khare, D. Shin, T.S. Yoo, M. Kim, T.D. Kang et al., Topotactic metal-insulator transition in epitaxial $\mathrm{SrFeO}_{\mathrm{x}}$ thin films. Adv. Mater. 29, 1606566 (2017). https://doi.org/10.1002/ adma.201606566

17. J.D. Ferguson, Y. Kim, L.F. Kourkoutis, A. Vodnick, A.R. Woll et al., Epitaxial oxygen getter for a brownmillerite phase transformation in manganite films. Adv. Mater. 23, 1226-1230 (2011). https://doi.org/10.1002/adma.201003581

18. J. Young, J.M. Rondinelli, Crystal structure and electronic properties of bulk and thin film brownmillerite oxides. Phys. Rev. B 92, 174111 (2015). https://doi.org/10.1103/PhysRevB. 92.174111

19. H. Jeen, W.S. Choi, J.W. Freeland, H. Ohta, C.U. Jung et al., Topotactic phase transformation of the brownmillerite $\mathrm{SrCoO}_{2.5}$ to the perovskite $\mathrm{SrCoO}_{3-\delta}$. Adv. Mater. 25, 36513656 (2013). https://doi.org/10.1002/adma.201300531

20. L. Cao, O. Petracic, P. Zakalek, A. Weber, U. Rücker et al., Reversible control of physical properties via an oxygen-vacancy-driven topotactic transition in epitaxial $\mathrm{La}_{0.7} \mathrm{Sr}_{0.3} \mathrm{MnO}_{3-\delta}$ thin films. Adv. Mater. 31, 1806183 (2019). https://doi.org/10.1002/adma.201806183

21. S. Chen, H. Zhou, X. Ye, Z. Chen, J. Zhao et al., Versatile and highly efficient controls of reversible topotactic metal-insulator transitions through proton intercalation. Adv. Funct. Mater. 29, 1907072 (2019). https://doi.org/10.1002/adfm.201907072

22. L. Yao, S. Majumdar, L. Äkäslompolo, S. Inkinen, Q.H. Qin et al., Electron-beam-induced perovskite-brownmillerite-perovskite structural phase transitions in epitaxial $\mathrm{La}_{2 / 3} \mathrm{Sr}_{1 / 3} \mathrm{MnO}_{3}$ films. Adv. Mater. 26, 2789-2793 (2014). https://doi.org/10. 1002/adma.201305656

23. L. Yao, S. Inkinen, S. van Dijken, Direct observation of oxygen vacancy-driven structural and resistive phase transitions in $\mathrm{La}_{2 / 3} \mathrm{Sr}_{1 / 3} \mathrm{MnO}_{3}$. Nat. Commun. 8, 14544 (2017). https://doi. org/10.1038/ncomms14544

24. K.T. Kang, B. Zhang, Y. Sharma, B. Paudel, H. Wang et al., Substrate oxygen sponge effect: A parameter for epitaxial manganite thin film growth. Appl. Phys. Lett. 117, 151601 (2020). https://doi.org/10.1063/5.0020426

25. S. Ning, Q. Zhang, C. Occhialini, R. Comin, X. Zhong et al., Voltage control of magnetism above room temperature in epitaxial $\mathrm{SrCo}_{1-\mathrm{x}} \mathrm{Fe}_{\mathrm{x}} \mathrm{O}_{3-\delta}$. ACS Nano 14, 8949-8957 (2020). https://doi.org/10.1021/acsnano.0c03750

26. D. Wang, L. Meng, L. Wei, P. Shi, Y. Chen et al., Reversible phase switching between antiferromagnetic $\mathrm{SrCoO}_{2.5}$ and ferromagnetic $\mathrm{SrCoO}_{3-\delta}$ by a flexible solid-state electrolyte gate. J. Magn. Magn. Mater. 496, 165926 (2020). https://doi.org/10. 1016/j.jmmm.2019.165926

27. L. He, D. Vanderbilt, First-principles study of oxygen-vacancy pinning of domain walls in $\mathrm{PbTiO}_{3}$. Phys. Rev. B 68, 134103 (2003). https://doi.org/10.1103/PhysRevB.68.134103

28. D. Lee, X. Gao, L. Sun, Y. Jee, J. Poplawsky et al., Colossal oxygen vacancy formation at a fluorite-bixbyite interface. Nat. Commun. 11, 1371 (2020). https://doi.org/10.1038/ s41467-020-15153-8

29. W. Zhang, L. Li, P. Lu, M. Fan, Q. Su et al., Perpendicular exchange-biased magnetotransport at the vertical interfaces 
in $\mathrm{La}_{07} \mathrm{Sr}_{0.3} \mathrm{MnO}_{3}: \mathrm{NiO}$ nanocomposites. ACS Appl. Mater. Interfaces 7, 21646-21651 (2015). https://doi.org/10.1021/ acsami.5b06314

30. W. Zhang, S. Cheng, C.M. Rouleau, K.P. Kelley, J. Keum et al., Unusual electrical conductivity driven by localized stoichiometry modification at vertical epitaxial interfaces. Mater. Horiz. 7, 3217-3225 (2020). https://doi.org/10.1039/D0MH0 1324B

31. J.L. MacManus-Driscoll, P. Zerrer, H. Wang, H. Yang, J. Yoon et al., Strain control and spontaneous phase ordering in vertical nanocomposite heteroepitaxial thin films. Nat. Mater. 7, 314-320 (2008). https://doi.org/10.1038/nmat2124

32. A. Chen, Z. Bi, Q. Jia, J.L. MacManus-Driscoll, H. Wang, Microstructure, vertical strain control and tunable functionalities in self-assembled, vertically aligned nanocomposite thin films. Acta Mater. 61, 2783-2792 (2013). https://doi.org/10. 1016/j.actamat.2012.09.072

33. J. Huang, J.L. MacManus-Driscoll, H. Wang, New epitaxy paradigm in epitaxial self-assembled oxide vertically aligned nanocomposite thin films. J. Mater. Res. 32, 4054-4066 (2017). https://doi.org/10.1557/jmr.2017.281

34. X. Sun, J.L. MacManus-Driscoll, H. Wang, Spontaneous ordering of oxide-oxide epitaxial vertically aligned nanocomposite thin films. Annu. Rev. Mater. Res. 50, 229-253 (2020). https://doi.org/10.1146/annurev-matsci-091719-112806

35. P. Gyanendra, A. Panchwanee, M. Kumar, K. Fritsch, R.J. Choudhary et al., Synthesis and characterization of vertically aligned $\mathrm{La}_{0.7} \mathrm{Sr}_{0.3} \mathrm{MnO}_{3}: \mathrm{NiO}$ nanocomposite thin films for spintronic applications. ACS Appl. Nano Mater. 4, 102-112 (2021). https://doi.org/10.1021/acsanm.0c02394
36. Y.J. Wu, Z.J. Wang, X.K. Ning, Q. Wang, W. Liu et al., Room temperature magnetoresistance properties in self-assembled epitaxial $\mathrm{La}_{0.7} \mathrm{Sr}_{0.3} \mathrm{MnO}_{3}: \mathrm{NiO}$ nanocomposite thin films. Mater. Res. Lett. 6, 489-494 (2018). https://doi.org/10.1080/ 21663831.2018.1482838

37. Y. Zhu, C. Ophus, J. Ciston, H. Wang, Interface lattice displacement measurement to $1 \mathrm{pm}$ by geometric phase analysis on aberration-corrected haadf stem images. Acta Mater. 61(15), 5646-5663 (2013). https://doi.org/10.1016/j.actamat.2013.06.006

38. J.F. Ding, O.I. Lebedev, S. Turner, Y.F. Tian, W.J. Hu et al., Interfacial spin glass state and exchange bias in manganite bilayers with competing magnetic orders. Phys. Rev. B 87, 054428 (2013). https://doi.org/10.1103/PhysRevB.87.054428

39. X. Ning, Z. Wang, Z. Zhang, Large, temperature-tunable lowfield magnetoresistance in $\mathrm{La}_{0.7} \mathrm{Sr}_{0.3} \mathrm{MnO}_{3}$ : $\mathrm{NiO}$ nanocomposite films modulated by microstructures. Adv. Funct. Mater. 24, 5393-5401 (2014). https://doi.org/10.1002/adfm.201400735

40. A. Chen, J.-M. Hu, P. Lu, T. Yang, W. Zhang et al., Role of scaffold network in controlling strain and functionalities of nanocomposite films. Sci. Adv. 2, e1600245 (2016). https:// doi.org/10.1126/sciadv. 1600245

41. D. Zhou, W. Sigle, M. Kelsch, H.-U. Habermeier, P.A. van Aken, Linking atomic structure and local chemistry at manganese-segregated antiphase boundaries in $\mathrm{ZrO}_{2}-\mathrm{La}_{2 / 3} \mathrm{Sr}_{1 / 3} \mathrm{MnO}_{3}$ thin films. Adv. Mater. Interfaces 2, 1500377 (2015). https:// doi.org/10.1002/admi.201500377

42. A. Chen, Q. Su, H. Han, E. Enriquez, Q. Jia, Metal oxide nanocomposites: A perspective from strain, defect, and interface. Adv. Mater. 31, 1803241 (2019). https://doi.org/10.1002/ adma.201803241 